Research Article

\title{
Pareto-Optimal Reinsurance Revisited: A Two-Stage Optimisation Procedure Approach
}

\author{
Ying Fang $\mathbb{D}^{1},{ }^{1}$ Lu Wang, ${ }^{2}$ and Zhongfeng $Q u^{3}$ \\ ${ }^{1}$ School of Mathematics and Statistics, Shandong Normal University, Jinan 250358, China \\ ${ }^{2}$ Caoxian No. 1 Middle School, Heze 274400, China \\ ${ }^{3}$ School of Mathematical Sciences, University of Jinan, Jinan 250022, China \\ Correspondence should be addressed to Ying Fang; fangying319@163.com
}

Received 16 June 2020; Accepted 21 July 2020; Published 29 September 2020

Guest Editor: Wenguang Yu

Copyright (C) 2020 Ying Fang et al. This is an open access article distributed under the Creative Commons Attribution License, which permits unrestricted use, distribution, and reproduction in any medium, provided the original work is properly cited.

In this paper, based on the Tail-Value-at-Risk (TVaR) measure, we revisit the Pareto-optimal reinsurance policies for the insurer and the reinsurer via a two-stage optimisation procedure. To reduce ex-post moral hazard, we assume that reinsurance contracts satisfy the principle of indemnity and the incentive compatible constraint which have been advocated by Huberman et al. (1983). We show that the Pareto-optimal reinsurance policy exists if the reinsurance premiums can be expressed as an integral form. The proposed class of premium principles encompasses the net premium principle, expected value premium principle, TVaR premium principle, generalized percentile premium principle, and so on. We further use the TVaR premium principle and the expected value premium principle as examples to illustrate the two-stage optimisation procedure by deriving explicitly the Paretooptimal reinsurance policies. We extend the results by Cai et al. (2017) when the expected value premium principle is replaced by the TVaR premium principle.

\section{Introduction}

The study of optimal reinsurance design has drawn great interest from both academics and practitioners since the seminal work by Borch [1] and Arrow [2]. There have been many important literatures and conclusions about this problem in the past few decades. For example, by minimizing the variance of the insurer's retained loss, Borch [1] showed that the stop-loss reinsurance is optimal under the expected value premium principle. Arrow [2] obtained that the optimal reinsurance policy is a stop-loss reinsurance strategy when the optimisation criterion is to maximize the expected utility function of the insurer. Both these results have been extended in a number of important directions. For example, Young [3] generalized Arrow's result under Wang's premium principle. Kaluszka [4] generalized Borch's result under mean-variance premium principles. Kaluszka and Okolewski [5] showed that the limited stop-loss and the truncated stop-loss are the optimal contracts under a number of criteria including the maximization of the expected utility, the stability, and the survival probability of the insurer for a fixed reinsurance premium calculated according to the maximal possible claims principle. Cai and Tan [6] developed two new optimisation criteria for deriving the optimal retentions by minimizing the Value-at-Risk $(\mathrm{VaR})$ and the conditional tail expectation (CTE) of the total risk of an insurer. In recent years, VaR and CTE have been used as optimisation criteria to study the optimal reinsurance strategy. For example, by minimizing VaR and CTE of an insurer's total cost, Cai et al. [7] derived the optimal reinsurance strategies in the set of increasing and convex ceded loss functions. The optimal reinsurance strategy depends on the confidence level of risk measurement, and it can be a stop-loss reinsurance strategy, a quota-share reinsurance strategy, or a change-loss reinsurance strategy. Bernard and Tian [8] provided alternative risk transfer mechanisms on the capital market when the optimal reinsurance is arranged under tail risk measures. Cheung [9] gave a geometric approach to revisit the optimal reinsurance problem and generalized the results in [7] by studying the 
VaR-minimization problem with Wang's premium principle. Chi and Tan [10] analyzed the VaR- and CVaR-based optimal reinsurance models over different classes of ceded loss functions with increasing generality. However, the above statements only consider the insurer, and from the point of view of the reinsurer, the optimal policy may not be optimal. For example, Vajda [11] showed that the optimal reinsurance strategy is a quota-share reinsurance instead of a stop-loss reinsurance when the optimisation criterion is to minimize the variance of the loss of the reinsurer. Thus, an optimal reinsurance contract for the insurer may not be optimal for the reinsurer and it might be unacceptable for the reinsurer. Then, an interesting question about optimal reinsurance is to design a reinsurance contract so that it considers the interests of both the insurer and the reinsurer.

Borch [1] first studied the optimal reinsurance strategy that consider the interests of both the insurer and the reinsurer. He discussed the optimal quota-share retention and stop-loss retention that maximize the product of the expected utility functions of the two parties' wealth. Kaishev [12] analyzed the optimal reinsurance contracts under which the finite horizon joint survival probability of the two parties is maximized. Under the general reinsurance principles, Cai et al. [13] took maximization of the joint survival probability and the joint profitable probability of both the insurer and the reinsurer as the optimisation criterion to give a sufficient condition of the optimal reinsurance existence. To maximize the joint survival probability of the insurer and the reinsurer, Fang and $\mathrm{Qu}[14]$ studied the optimal policy of combination of quota-share reinsurance and stop-loss reinsurance. Fang et al. [15] studied the optimal reinsurance models from the perspective of both the insurer and the reinsurer by minimizing their total costs under the criteria of the loss function which is defined by the joint Value-at-Risk. Cai et al. [16] studied the optimal reinsurance strategy, which was based on the minimum convex combination of the $\mathrm{VaR}$ of the insurer and the reinsurer under two types of constraints. Lo [17] discussed the generalized problems in [16] by using the Neyman-Pearson approach. Based on the optimal reinsurance strategy in [16], Jiang et al. [18] proved that the optimal reinsurance strategy is a Pareto-optimal reinsurance policy and gave optimal reinsurance strategies using the geometric method. Cai et al. [19] studied the Pareto optimality of reinsurance arrangements under general model settings and obtained the explicit forms of the Pareto-optimal reinsurance contracts under the TVaR measure and the expected value premium principle. Jiang et al. [20] studied the optimal reinsurance with constraints under the distortion risk measure. By the geometric approach, Fang et al. [21] studied Pareto-optimal reinsurance policies under general premium principles and gave the explicit parameters of the optimal ceded loss functions under the Dutch premium principle and Wang's premium principle. Lo and Tang [22] characterized the set of Pareto-optimal reinsurance policies analytically and visualized the insurer-reinsurer trade-off structure geometrically. Huang and Yin [23] studied two classes of optimal reinsurance models from perspectives of both insurers and reinsurers by minimizing their convex combination where the risk is measured by a distortion risk measure and the premium is given by a distortion premium principle.

In this paper, based on the TVaR measure, we revisit the Pareto-optimal reinsurance policies for the insurer and the reinsurer via a two-stage optimisation procedure which was proposed by Asimit et al. [24]. To reduce ex-post moral hazard, we assume that reinsurance contracts satisfy the principle of indemnity and the incentive compatible constraint which have been advocated by Huberman et al. [25]. We first show that the Pareto-optimal reinsurance policy exists if the reinsurance premiums can be expressed as an integral form such as (10). We emphasize that there are many premium principles which satisfy this property such as the net premium principle, expected value premium principle, TVaR premium principle, and generalized percentile premium principle. Then, we take the TVaR premium principle and the expected value premium principle as examples to illustrate the two-stage optimisation procedure by deriving explicitly the parameters of the Pareto-optimal reinsurance policies.

It is worth noting that the Pareto-optimal reinsurance contracts under the TVaR measure and the expected value premium principle has been obtained in [19]. We reexamine this problem for two reasons. First, it should be emphasized that the results can be achieved by using a different approach based on a two-stage optimisation procedure. Second, and more importantly, the two-stage optimisation procedure is more intuitive and can analyze Pareto-optimal reinsurance policies with other reinsurance premium principles.

The remaining arrangements of this paper are as follows. In Section 2, we introduce some definitions and model formulation and then we show that the Pareto-optimal reinsurance policy exists if the reinsurance premiums can be expressed as an integral form such as (10). Based on the TVaR measure, we obtain the Pareto-optimal policies under the TVaR premium principle and the expected value premium principle in Section 3. In Section 4, we give illustrative numerical examples. Section 5 concludes the paper. Finally, all the proofs are given in the Appendix.

\section{Model Formulation}

Let $X$ be the amount of loss faced by the insurer in a given time period. Suppose that $X$ is a nonnegative random variable with a distribution function $F_{X}(x)=\mathrm{P}\{X \leq x\}$ and survival function $S_{X}(x)=1-F_{X}(x)$. In addition, the value of the right endpoint $X_{F}$ of the distribution function $F_{X}(x)$ can be either finite or infinite, where $X_{F}:=\inf \{z: F(z)=1\}$. Under a reinsurance arrangement, $R(X)$ and $I_{R}(X)$ represent the ceded loss and the retained loss of the insurer, respectively, where $I_{R}(X)=X-R(X)$. Functions $R(x)$ and $I_{R}(x)$ are called the ceded loss function and the retained loss function. The principle of indemnity, which is widely used in insurance and reinsurance, requires the indemnity to be nonnegative and less than the initial loss. Mathematically, we should have $0 \leq R(x) \leq x$. Let $\pi(R(X))$ be the reinsurance premium. Under such a setting, the total losses of the insurer and the reinsurer are $M_{R}:=X-R(X)+\pi(R(X))$ and $N_{R}:=R(X)-\pi(R(X))$, respectively. 
In this paper, besides the principle of indemnity, we also assume that reinsurance contracts satisfy the incentive compatible constraint which has been advocated by Huberman et al. [25] to reduce ex-post moral hazard. This means that the more the realized loss, the more paid by both the insurer and the reinsurer. Mathematically, this implies that both the ceded loss function and the retained loss function should be increasing. Therefore, throughout the paper, we assume that the admissible set of ceded loss functions is given by

$$
\begin{aligned}
\mathscr{F}= & \{R(x): 0 \leq R(x) \leq x, \text { both } R(x) \\
& \text { and } \left.I_{R}(x) \text { are increasing functions }\right\} .
\end{aligned}
$$

It was shown by Chi and Tan [10] that all functions $R(x) \in \mathscr{F}$ are Lipschitz continuous and differentiable almost everywhere.

In insurance and finance, risk measures such as VaR and TVaR have been widely used for quantifying risks. Now, we give a brief description of $\mathrm{VaR}$ and $\mathrm{TVaR}$ measures:

Definition 1 (VaR). For a random variable $X, \mathrm{VaR}$ is defined as

$$
\operatorname{VaR}_{p}(X):=\inf \{\{x \in R: \mathrm{P}(X \leq x) \geq p\},
$$

where $0<p<1$ represents a confidence level of the loss variable $X$.

Definition 2 (TVaR). For a random variable $X$, TVaR is defined as

$$
\begin{aligned}
\operatorname{TVaR}_{p}(X):= & \frac{1}{1-p} \int_{p}^{1} \operatorname{VaR}_{s}(X) \mathrm{d} s=\operatorname{VaR}_{p}(X) \\
& +\frac{1}{1-p} E\left(X-\operatorname{VaR}_{p}(X)\right)_{+}
\end{aligned}
$$

where $0<p<1$ represents a confidence level of the loss variable $X$.

\section{Remark 1}

(1) By the definitions of the VaR and the TVaR, distinctly, $\mathrm{TVaR}_{p}$ evaluates the expected loss amount incurred among the worst $(1-p) \%$ scenarios under a confidence level $p$. Therefore, the TVaR represents a more precise risk measurement than the VaR.

(2) When $1-p \geq S_{X}(0)$, we have $\operatorname{VaR}_{p}(X)=0$. Therefore, in order to avoid a trivial case, we assume that $1-p \in\left(0, S_{X}(0)\right)$.

In this paper, we assume that the confidence levels of the insurer and the reinsurer are possibly different. Let $\alpha_{c}$ and $\alpha_{r}$ denote the confidence levels of the insurer and the reinsurer, respectively. Therefore, the total loss of the insurer and the reinsurer under the TVaR measure is

$$
\begin{aligned}
& \operatorname{TVaR}_{\alpha_{c}}\left(M_{R}\right)=\operatorname{VaR}_{\alpha_{c}}\left(M_{R}\right)+\frac{1}{1-\alpha_{c}} E\left(M_{R}-\operatorname{VaR}_{\alpha_{c}}\left(M_{R}\right)\right)_{+}, \\
& \operatorname{TVaR}_{\alpha_{r}}\left(N_{R}\right)=\operatorname{VaR}_{\alpha_{r}}\left(N_{R}\right)+\frac{1}{1-\alpha_{r}} E\left(N_{R}-\operatorname{VaR}_{\alpha_{r}}\left(N_{R}\right)\right)_{+} .
\end{aligned}
$$

Next, we study Pareto-optimal reinsurance policies whereby the risk is measured by the TVaR. For our model, a reinsurance policy with the ceded loss function $R^{*}(x)$ is called Pareto optimal if there is no other admissible ceded loss function $R \in \mathscr{F}$ such that $\operatorname{TVaR}_{\alpha_{c}}\left(M_{R}\right) \leq \operatorname{TVaR}_{\alpha_{c}}\left(M_{R^{*}}\right)$ and $\operatorname{TVaR}_{\alpha_{r}}\left(N_{R}\right) \leq \operatorname{TVaR}_{\alpha_{r}}\left(N_{R^{*}}\right)$ and at least one of the inequalities is strict. A general approach to identify Paretooptimal reinsurance policies is to minimize a convex combination of the TVaRs of the two parties. The result can be found in [18-20].

Proposition 1. All Pareto-optimal reinsurance policies $R$ in $\mathscr{F}$ can be determined by solving the problem:

$$
\min _{R \in \mathscr{F}}\left\{\beta \operatorname{TVaR}_{\alpha_{c}}\left(M_{R}\right)+(1-\beta) \operatorname{TVaR}_{\alpha_{r}}\left(N_{R}\right)\right\},
$$

where $\beta \in[0,1]$.

In view of Proposition 1, throughout the rest of this paper, we only need to determine optimal reinsurance policies by solving the optimisation problem (5). Define

$$
V(R)=\beta \operatorname{TVaR}_{\alpha_{c}}\left(M_{R}\right)+(1-\beta) \operatorname{TVaR}_{\alpha_{r}}\left(N_{R}\right) .
$$

Then, by translation invariance and comonotonic additivity of TVaR, we have

$$
\begin{aligned}
V(R)= & \beta \operatorname{TVaR}_{\alpha_{c}}(X)-\beta \operatorname{TVaR}_{\alpha_{c}}(R(X)) \\
& +(1-\beta) \operatorname{TVaR}_{\alpha_{r}}(R(X))+(2 \beta-1) \pi(R(X)) .
\end{aligned}
$$

Therefore, the optimisation problem (5) becomes

$$
\min _{R \in \mathscr{F}} H(R),
$$

where

$$
\begin{aligned}
H(R)= & -\beta \operatorname{TVaR}_{\alpha_{c}}(R(X))+(1-\beta) \operatorname{TVaR}_{\alpha_{r}}(R(X)) \\
& +(2 \beta-1) \pi(R(X)) .
\end{aligned}
$$

In this paper, we determine the Pareto-optimal reinsurance policies via a two-stage optimisation procedure which was developed in [24]. The first stage is solving an infinite-dimensional problem, while the second stage becomes a classical constrained optimisation problem. The first stage can be solved as shown in Proposition 1 in [24], and we now present it as a lemma.

Lemma 1. Let $f(\cdot)$ be a real-valued function defined on $\left[s_{1}, s_{2}\right]$ with $0 \leq s_{1} \leq s_{2} \leq 1$. Then, 


$$
\begin{array}{ll}
\min _{R \in \mathscr{F}} & \int_{s_{1}}^{s_{2}} f(s) R\left(\operatorname{VaR}_{s}(x)\right) \mathrm{d} s, \\
\text { subjectto } & R\left(\operatorname{VaR}_{s_{1}}(X)\right)=\xi_{1}, \\
& R\left(\operatorname{VaR}_{s_{2}}(X)\right)=\xi_{2},
\end{array}
$$

is uniquely solved by

$$
R^{*}\left(X ; \xi_{1}, \xi_{2}\right)=\left\{\begin{array}{l}
\left(X-\operatorname{VaR}_{s_{1}}(X)+\xi_{1}\right) \wedge \xi_{2}, \quad \text { if } f(s)<0 \text { for all } s_{1} \leq s \leq s_{2}, \\
\xi_{1}+\left(X-\operatorname{VaR}_{s_{2}}(X)+\xi_{2}-\xi_{1}\right)_{+}, \quad \text { if } f(s)>0 \text { for all } s_{1} \leq s \leq s_{2},
\end{array}\right.
$$

where $\left(\xi_{1}, \xi_{2}\right)$ are some constants such that $0 \leq \xi_{2}-$ $\xi_{1} \leq \operatorname{VaR}_{s_{2}}(X)-\operatorname{VaR}_{s_{1}}(X)$.

Note that

$$
\begin{aligned}
H(R)= & \int_{\alpha_{c}}^{1} \frac{-\beta}{1-\alpha_{c}} R\left(\operatorname{VaR}_{s}(X)\right) \mathrm{d} s \\
& +\int_{\alpha_{r}}^{1} \frac{1-\beta}{1-\alpha_{r}} R\left(\operatorname{VaR}_{s}(X)\right) \mathrm{d} s+(2 \beta-1) \pi(R(X))
\end{aligned}
$$

By Lemma 1, we know that the Pareto-optimal reinsurance policy exists if the reinsurance premiums $\pi(R(X))$ can be expressed as an integral form such as (10). Next, we give several premium principles which satisfy this property:

(1) Net premium principle: $\pi(X)=E(X)$. Since $R(x)$ is a nondecreasing continuous function, then $\pi(R(X))=\int_{0}^{1} R\left(\operatorname{VaR}_{s}(X)\right) \mathrm{d} s$.

(2) Expected value premium principle: $\pi(X)=(1+$ $\theta) E(X)$, where $\theta \in[0,1]$ is a safety loading coefficient. Therefore, $\pi(R(X))=(1+\theta) \int_{0}^{1} R\left(\mathrm{VaR}_{s}\right.$ $(X)) \mathrm{d} s$.

(3) $\mathrm{TVaR}$ premium principle: $\pi(X)=(1+\theta /$ $1-\alpha) \int_{\alpha}^{1} \operatorname{VaR}_{s}(X) \mathrm{d} s$, where $\alpha \in[0,1)$ is a confidence level and $\theta \in[0,1]$ is a safety loading coefficient. Since $R(x)$ is a nondecreasing continuous function, then $\pi(R(X))=(1+\theta / 1-\alpha) \int_{\alpha}^{1} R\left(\operatorname{VaR}_{s}(X)\right) \mathrm{d} s$.

(4) Generalized percentile premium principle: $\pi(X)=$ $E(X)+\beta\left\{F_{X}^{-1}(1-p)-E(X)\right\} \quad$ with $\quad 0<\beta, p<1$. Since $F_{X}^{-1}(1-p)=\operatorname{VaR}_{1-p}(X)$, then $\pi(R(X))=$ $(1-\beta) E[R(X)]+\beta R\left(\operatorname{VaR}_{1-p}(X)\right)$.

In the following sections, we take the TVaR premium principle and the expected value premium principle as examples to illustrate the two-stage optimisation procedure.

\section{Pareto-Optimal Reinsurance Policy}

In this section, we determine the Pareto-optimal reinsurance policies under the TVaR premium principle and the expected value premium principle. The TVaR premium principle was first proposed by Young [26]. It can be viewed as an extended version of the expected value premium principle, that is, letting $\alpha=0$ gives the expected value premium principle.
3.1. Pareto-Optimal Reinsurance Policies under TVaR Principle. Under the TVaR premium principle, the optimisation problem (8) becomes

$$
\min _{R \in \mathscr{F}} H(R),
$$

where $H(R)=-\beta \operatorname{TVaR}_{\alpha}(R(X))+(1-\beta) \operatorname{TVaR}_{\alpha}(R(X))+$ $(2 \beta-1)(1+\theta) \operatorname{TVaR}_{\alpha}(R(X))$. From the mathematical point of view, the confidence level $\alpha$ can be larger than confidence levels $\alpha_{c}$ and $\alpha_{r}$. However, $\alpha$ is usually smaller while $\alpha_{c}$ and $\alpha_{r}$ are usually larger in practice. So, we assume further $\alpha<\min \left\{\alpha_{c}, \alpha_{r}\right\}$ to avoid complex and lengthy discussions in this section.

For simplicity, we define the following notations:

$$
\begin{aligned}
a & =\operatorname{VaR}_{\alpha}(X), \\
a_{c} & =\operatorname{VaR}_{\alpha_{c}}(X), \\
a_{r} & =\operatorname{VaR}_{\alpha_{r}}(X), \\
\xi & =R\left(\operatorname{VaR}_{\alpha}(X)\right), \\
\xi_{c} & =R\left(\operatorname{VaR}_{\alpha_{c}}(X)\right), \\
\xi_{r} & =R\left(\operatorname{VaR} \alpha_{r}(X)\right), \\
m & =\frac{(2 \beta-1)(1+\theta)}{1-\alpha}-\frac{\beta}{1-\alpha_{c}}, \\
n & =\frac{(2 \beta-1)(1+\theta)}{1-\alpha}+\frac{1-\beta}{1-\alpha_{r}}, \\
s(\beta) & =1-\frac{\beta-1}{m}, \\
t(\beta) & =1-\frac{\beta}{n} .
\end{aligned}
$$

Next, we divide our discussion into three cases: (1) $a_{c}<a_{r}$; (2) $a_{r}<a_{c}$; (3) $a_{c}=a_{r}$. Then, we obtain the following three theorems.

Theorem 1. Under the condition $a_{c}<a_{r}$, the Pareto-optimal reinsurance policies are given as follows: 
(1) If $0 \leq \beta<1 / 2$ and $\left(\beta-1 / 1-\alpha_{r}\right)<m$, then

$R^{*}(x)=\left\{\begin{array}{l}x \wedge \operatorname{VaR}_{s(\beta)}(X), \quad \text { when }(1+\theta)\left(1-\alpha_{c}\right)>1-\alpha, \\ x \wedge \operatorname{VaR}_{(\theta+\alpha / 1+\theta)}(X), \quad \text { when }(1+\theta)\left(1-\alpha_{c}\right) \leq 1-\alpha .\end{array}\right.$

(2) If $0 \leq \beta<1 / 2$ and $\left(\beta-1 / 1-\alpha_{r}\right)>m$, then $R^{*}(x)=x$.

(3) If $0 \leq \beta<1 / 2$ and $\left(\beta-1 / 1-\alpha_{r}\right)=m$, then $R^{*}(x)=$ $x I_{\left\{a \leq x \leq a_{r}\right\}}+R(x) I_{\left\{x>a_{r}\right\}}$, where $R(x)$ is any increasing 1-Lipschitz continuous function such that $R^{*}(x) \in \mathscr{F}$.
(4) If $\beta=1 / 2$, then $R^{*}(x)=R(x) I_{\left\{a \leq x \leq a_{c}\right\}}+u_{1} I_{\left\{x>a_{c}\right\}}$, where $u_{1}$ is an arbitrary constant in $\left[u, a_{c}-a+u\right], u$ is an arbitrary constant in $[0, a]$, and $R(x)$ is any increasing 1-Lipschitz continuous function such that $R^{*}(x) \in \mathscr{F}$.

(5) If $1 / 2<\beta \leq 1$ and $m>0$, then $R^{*}(x)=0$.

(6) If $1 / 2<\beta<1$ and $\left(\beta-1 / 1-\alpha_{r}\right)<m<0$, then

$$
R^{*}(x)=\left\{\begin{array}{l}
0, \quad \text { when }(1+\theta)\left(1-\alpha_{c}\right) \geq 1-\alpha, \\
\left(x-\operatorname{VaR}_{(\theta+\alpha / 1+\theta)}(X)\right)_{+} \wedge\left(\operatorname{VaR}_{s(\beta)}(X)-\operatorname{VaR}_{(\theta+\alpha / 1+\theta)}(X)\right), \quad \text { when }(1+\theta)\left(1-\alpha_{c}\right)<1-\alpha .
\end{array}\right.
$$

(7) If $1 / 2<\beta \leq 1$ and $m<\left(\beta-1 / 1-\alpha_{r}\right)$, then $R^{*}(x)=$ $\left(x-\operatorname{VaR}_{(\theta+\alpha / 1+\theta)}(X)\right)_{+}$.

(8) If $1 / 2<\beta<1$ and $m=\left(\beta-1 / 1-\alpha_{r}\right)$, then $R^{*}(x)=$ $\left(x-\operatorname{VaR}_{(\theta+\alpha / 1+\theta)}(X)\right)_{+} I_{\left\{a \leq x \leq a_{r}\right\}}+R(x) I_{\left\{x>a_{r}\right\}}$. where $R(x)$ is any increasing 1 -Lipschitz continuous function such that $R^{*}(x) \in \mathscr{F}$.

(9) If $1 / 2<\beta<1$ and $m=0$, then $R^{*}(x)=0$.

(10) If $\beta=1$ and $(1+\theta)\left(1-\alpha_{c}\right)=1-\alpha$, then $R^{*}(x)=$ $R(x) I_{\left\{x>a_{c}\right\}}$, where $R(x)$ is any increasing 1-Lipschitz continuous function such that $R^{*}(x) \in \mathscr{F}$.
Theorem 2. Under the condition $a_{r}<a_{c}$, the Pareto-optimal reinsurance policies are given as follows:

(1) If $\beta=0$ and $(1+\theta)\left(1-\alpha_{r}\right)=1-\alpha$, then $R^{*}(x)=$ $x I_{\left\{a \leq x \leq a_{r}\right\}}+R(x) I_{\left\{x>a_{r}\right\}}$, where $R(x)$ is any increasing 1-Lipschitz continuous function such that $R^{*}(x) \in \mathscr{F}$.

(2) If $0 \leq \beta<1 / 2$ and $n>\left(\beta / 1-\alpha_{c}\right)$, then $R^{*}(x)=$ $x \wedge \operatorname{VaR}_{(\theta+\alpha / 1+\theta)}(X)$.

(3) If $0<\beta<1 / 2$ and $0<n<\left(\beta / 1-\alpha_{c}\right)$, then

$$
R^{*}(x)=\left\{\begin{array}{l}
x \wedge \operatorname{VaR}_{(\theta+\alpha / 1+\theta)}(X)+\left(x-\operatorname{VaR}_{t(\beta)}(X)\right)_{+}, \quad \text { when }(1+\theta)\left(1-\alpha_{r}\right)<1-\alpha, \\
x, \quad \text { when }(1+\theta)\left(1-\alpha_{r}\right) \geq 1-\alpha .
\end{array}\right.
$$

(4) If $0<\beta<1 / 2$ and $n=\left(\beta / 1-\alpha_{c}\right)$, then $R^{*}(x)=$ $\left(x \wedge V a R_{(\theta+\alpha / 1+\theta)}(X)\right) I_{\left\{a \leq x \leq a_{c}\right\}}+R(x) I_{\left\{x>a_{c}\right\}}$, where $R(x)$ is any increasing 1-Lipschitz continuous function such that $R^{*}(x) \in \mathscr{F}$.

(5) If $0 \leq \beta<1 / 2$ and $n<0$, then $R^{*}(x)=x$.

(6) If $0<\beta<1 / 2$ and $n=0$, then $R^{*}(x)=$ $x I_{\left\{a \leq x \leq a_{r} \text { or } x>a_{c}\right\}}+R(x) I_{\left\{a_{r}<x \leq a_{c}\right\}}$, where $R(x)$ is any increasing 1-Lipschitz continuous function such that $R^{*}(x) \in \mathscr{F}$.

(7) If $\beta=1 / 2$, then $R^{*}(x)=R(x) I_{\left\{a \leq x \leq a_{r}\right\}}+\left(x-a_{r}+u_{2}\right)$ $I_{\left\{x>a_{r}\right\}}$, where $u_{2}$ is an arbitrary constant in $\left[u, a_{r}-a+u\right], u$ is an arbitrary constant in $[0, a]$, and $R(x)$ is any increasing 1-Lipschitz continuous function such that $R^{*}(x) \in \mathscr{F}$.

(8) If $1 / 2<\beta \leq 1$ and $n>\left(\beta / 1-\alpha_{c}\right)$, then $R^{*}(x)=0$.

(9) If $1 / 2<\beta \leq 1$ and $0<n<\left(\beta / 1-\alpha_{c}\right)$, then
$R^{*}(x)=\left\{\begin{array}{l}\left(x-\operatorname{VaR}_{(\theta+\alpha / 1+\theta)}(X)\right)_{+}, \quad \text { when }(1+\theta)\left(1-\alpha_{r}\right) \leq 1-\alpha, \\ \left(x-\operatorname{VaR}_{t(\beta)}(X)\right)_{+}, \quad \text { when }(1+\theta)\left(1-\alpha_{r}\right)>1-\alpha .\end{array}\right.$

(10) If $1 / 2<\beta \leq 1$ and $n=\left(\beta / 1-\alpha_{c}\right)$, then $R^{*}(x)=$ $R(x) I_{\left\{x>a_{c}\right\}}$, where $R(x)$ is any increasing 1-Lipschitz continuous function such that $R^{*}(x) \in \mathscr{F}$.

Theorem 3. Under the condition $a_{c}=a_{r}$, the Pareto-optimal reinsurance policies are given as follows:

(1) If $0 \leq \beta<1 / 2$, then

$$
R^{*}(x)=\left\{\begin{array}{l}
x, \quad \text { when }(1+\theta)\left(1-\alpha_{c}\right)>1-\alpha, \\
x I_{\left\{a \leq x \leq a_{c}\right\}}+R(x) I_{\left\{x>a_{c}\right\}}, \quad \text { when }(1+\theta)\left(1-\alpha_{c}\right)=1-\alpha, \\
x \wedge \operatorname{VaR}_{(\theta+\alpha / 1+\theta)}(X), \quad \text { when }(1+\theta)\left(1-\alpha_{c}\right)<1-\alpha,
\end{array}\right.
$$


where $R(x)$ is any increasing 1-Lipschitz continuous function such that $R^{*}(x) \in \mathscr{F}$.

(2) If $\beta=1 / 2$, the objective function is identical to 0 and the problem is trivial.

(3) If $1 / 2<\beta \leq 1$, then

$$
R^{*}(x)=\left\{\begin{array}{l}
0, \quad \text { when }(1+\theta)\left(1-\alpha_{c}\right)>1-\alpha, \\
R(x) I_{\left\{x>a_{c}\right\}}, \quad \text { when }(1+\theta)\left(1-\alpha_{c}\right)=1-\alpha, \\
\left(x-\operatorname{VaR}_{(\theta+\alpha / 1+\theta)}(X)\right)_{+}, \quad \text { when }(1+\theta)\left(1-\alpha_{c}\right)<1-\alpha,
\end{array}\right.
$$

where $R(x)$ is any increasing 1-Lipschitz continuous function such that $R^{*}(x) \in \mathscr{F}$.

3.2. Pareto-Optimal Reinsurance Policies under Expected Value Principle. In this section, we reexamine an optimal reinsurance problem studied in [19], in which the objective is to find the optimal reinsurance contracts that minimize the TVaR of the total risk exposure under the expected value premium principle. We provide a more intuitive approach to solve the problem by using a two-stage optimisation method. Under the expected value principle, the optimisation problem (5) becomes

$$
\begin{aligned}
& \min _{R \in \mathscr{F}}\left\{-\beta \operatorname{TVaR}_{\alpha_{c}}(R(X))+(1-\beta) \operatorname{TVaR}_{\alpha_{r}}(R(X))\right. \\
& \quad+(2 \beta-1)(1+\theta) E(R(X))\} .
\end{aligned}
$$

For simplicity, we define the following notations:

$$
\begin{aligned}
m_{0} & =(2 \beta-1)(1+\theta)-\frac{\beta}{1-\alpha_{c}}, \\
n_{0} & =(2 \beta-1)(1+\theta)+\frac{1-\beta}{1-\alpha_{r}}, \\
s_{0}(\beta) & =1-\frac{\beta-1}{m_{0}}, \\
t_{0}(\beta) & =1-\frac{\beta}{n_{0}} .
\end{aligned}
$$

Theorem 4. Under the condition $a_{c}<a_{r}$, the Pareto-optimal reinsurance policies are given as follows:

(1) If $0 \leq \beta<1 / 2$ and $\left(\beta-1 / 1-\alpha_{r}\right)<m_{0}<0$, then

$$
R^{*}(x)=\left\{\begin{array}{l}
x \wedge \operatorname{VaR}_{s_{0}(\beta)}(X), \quad \text { when }(1+\theta)\left(1-\alpha_{c}\right)>1, \\
0, \quad \text { when } S_{X}(0) \leq \theta^{*} \text { and }(1+\theta)\left(1-\alpha_{c}\right) \leq 1, \\
x \wedge \operatorname{VaR}_{(\theta / 1+\theta)}(X), \quad \text { when } S_{X}(0)>\theta^{*} \text { and }(1+\theta)\left(1-\alpha_{c}\right) \leq 1 .
\end{array}\right.
$$

(2) If $0 \leq \beta<1 / 2$ and $m_{0}<\left(\beta-1 / 1-\alpha_{r}\right)$, then $R^{*}(x)=x$.

(3) If $0 \leq \beta<1 / 2$ and $\left(\beta-1 / 1-\alpha_{r}\right)=m_{0}$, then $R^{*}(x)=x I_{\left\{x \leq a_{r}\right\}}+R(x) I_{\left\{x>a_{r}\right\}}$, where $R(x)$ is an increasing 1-Lipschitz continuous function such that $R^{*}(x) \in \mathscr{F}$.
(4) If $\beta=1 / 2$, then $R^{*}(x)=R(x) I_{\left\{x \leq a_{c}\right\}}+u_{3} I_{\left\{x>a_{c}\right\}}$, where $R(x)$ is an increasing 1-Lipschitz continuous function such that $R^{*}(x) \in \mathscr{F}$ and $u_{3} \in\left[0, a_{c}\right]$.

(5) If $1 / 2<\beta \leq 1$ and $m_{0}>0$, then $R^{*}(x)=0$.

(6) If $1 / 2<\beta<1$ and $\left(\beta-1 / 1-\alpha_{r}\right)<m_{0}<0$, then

$$
R^{*}(x)=\left\{\begin{array}{l}
0, \quad \text { when }(1+\theta)\left(1-\alpha_{c}\right) \geq 1, \\
x \wedge \operatorname{VaR}_{s_{0}(\beta)}(X), \quad \text { when } S_{X}(0) \leq \theta^{*} \text { and }(1+\theta)\left(1-\alpha_{c}\right)<1, \\
\left(x-\operatorname{VaR}_{(\theta / 1+\theta)}(X)\right)_{+} \wedge\left(\operatorname{VaR}_{s_{0}(\beta)}(X)-\operatorname{VaR}_{(\theta / 1+\theta)}(X)\right), \quad \text { when } S_{X}(0)>\theta^{*} \text { and }(1+\theta)\left(1-\alpha_{c}\right)<1 .
\end{array}\right.
$$

(7) If $1 / 2<\beta \leq 1$ and $m_{0}<\left(\beta-1 / 1-\alpha_{r}\right)$, then

$R^{*}(x)=\left\{\begin{array}{l}x, \quad \text { when } S_{X}(0) \leq \theta^{*}, \\ \left(x-\operatorname{VaR}_{(\theta / 1+\theta)}(X)\right)_{+}, \quad \text { when } S_{X}(0)>\theta^{*} .\end{array}\right.$

(8) If $1 / 2<\beta<1$ and $m_{0}=\left(\beta-1 / 1-\alpha_{r}\right)$, then

$$
R^{*}(x)=\left\{\begin{array}{l}
x I_{\left\{x \leq a_{r}\right\}}+R(x) I_{\left\{x>a_{r}\right\}}, \quad \text { when } S_{X}(0) \leq \theta^{*}, \\
\left(x-\operatorname{VaR}_{(\theta / 1+\theta)}(X)\right)_{+} I_{\left\{x \leq a_{r}\right\}}+R(x) I_{\left\{x>a_{r}\right\}}, \quad \text { when } S_{X}(0)>\theta^{*},
\end{array}\right.
$$

where $R(x)$ is an increasing 1-Lipschitz continuous function such that $R^{*}(x) \in \mathscr{F}$.

(9) If $1 / 2<\beta<1$ and $m_{0}=0$, then $R^{*}(x)=0$. 
(10) If $\beta=1$ and $(1+\theta)\left(1-\alpha_{c}\right)=1$, then $R^{*}(x)=$ $R(x) I_{\left\{x>a_{c}\right\}}$, where $R(x)$ is an increasing 1-Lipschitz continuous function such that $R^{*}(x) \in \mathscr{F}$.

Theorem 5. Under the condition $a_{r}<a_{c}$, the Pareto-optimal reinsurance policies are given as follows:

(1) If $\beta=0$ and $(1+\theta)\left(1-\alpha_{r}\right)=1$, then $R^{*}(x)=$ $x I_{\left\{x \leq a_{r}\right\}}+R(x) I_{\left\{x>a_{r}\right\}}$, where $R(x)$ is an increasing 1Lipschitz continuous function such that $R^{*}(x) \in \mathscr{F}$.
(2) If $0 \leq \beta<1 / 2$ and $n_{0}>\left(\beta / 1-\alpha_{c}\right)$, then

$R^{*}(x)=\left\{\begin{array}{l}0, \quad \text { when } S_{X}(0) \leq \theta^{*}, \\ x \wedge \operatorname{VaR}_{(\theta / 1+\theta)}(X), \quad \text { when } S_{X}(0)>\theta^{*} .\end{array}\right.$

(3) If $0<\beta<1 / 2$ and $0<n_{0}<\left(\beta / 1-\alpha_{c}\right)$, then

$$
R^{*}(x)=\left\{\begin{array}{l}
x, \quad \text { when }(1+\theta)\left(1-\alpha_{r}\right) \geq 1, \\
\left(x-\operatorname{VaR}_{t_{0}(\beta)}(X)\right)_{+}, \quad \text { when } S_{X}(0) \leq \theta^{*} \text { and }(1+\theta)\left(1-\alpha_{r}\right)<1, \\
x \wedge \operatorname{VaR}_{(\theta / 1+\theta)}(X)+\left(x-\operatorname{VaR}_{t_{0}(\beta)}(X)\right)_{+}, \quad \text { when } S_{X}(0)>\theta^{*} \text { and }(1+\theta)\left(1-\alpha_{r}\right)<1 .
\end{array}\right.
$$

(4) If $0<\beta<1 / 2$ and $n_{0}=\left(\beta / 1-\alpha_{c}\right)$, then

$$
R^{*}(x)=\left\{\begin{array}{l}
R(x) I_{\left\{x>a_{c}\right\}}, \quad \text { when } S_{X}(0) \leq \theta^{*}, \\
\left\{x \wedge \operatorname{VaR}_{(\theta / 1+\theta)}(X)\right\} I_{\left\{x \leq a_{c}\right\}}+R(x) I_{\left\{x>a_{c}\right\}}, \quad \text { when } S_{X}(0)>\theta^{*},
\end{array}\right.
$$

where $R(x)$ is an increasing 1-Lipschitz continuous function such that $R^{*}(x) \in \mathscr{F}$.

(5) If $0 \leq \beta<1 / 2$ and $n_{0}<0$, then $R^{*}(x)=x$.

(6) If $0<\beta<1 / 2$ and $n_{0}=0$, then $R^{*}(x)=$ $x I_{\left\{x \leq a_{r} \text { or } x>a_{c}\right\}}+R(x) I_{\left\{a_{r}<x \leq a_{c}\right\}}$, where $R(x)$ is an increasing 1-Lipschitz continuous function such that $R^{*}(x) \in \mathscr{F}$.
(7) If $\beta=1 / 2$, then $R^{*}(x)=R(x) I_{\left\{x \leq a_{r}\right\}^{+}} \quad\left(x-a_{r}+\right.$ $\left.u_{4}\right) I_{\left\{x>a_{r}\right\}}$, where $R(x)$ is an increasing 1-Lipschitz continuous function such that $R^{*}(x) \in \mathscr{F}$ and $u_{4} \in\left[0, a_{r}\right]$.

(8) If $1 / 2<\beta \leq 1$ and $n_{0}>\left(\beta / 1-\alpha_{c}\right)$, then $R^{*}(x)=0$.

(9) If $1 / 2<\beta \leq 1$ and $0<n_{0}<\left(\beta / 1-\alpha_{c}\right)$, then

$$
R^{*}(x)=\left\{\begin{array}{l}
\left(x-\operatorname{VaR}_{t_{0}(\beta)}(X)\right)_{+}, \quad \text { when }(1+\theta)\left(1-\alpha_{r}\right) \geq 1, \\
x, \quad \text { when } S_{X}(0) \leq \theta^{*} \text { and }(1+\theta)\left(1-\alpha_{r}\right)<1, \\
\left(x-\operatorname{VaR}_{(\theta / 1+\theta)}(X)\right)_{+}, \quad \text { when } S_{X}(0)>\theta^{*} \text { and }(1+\theta)\left(1-\alpha_{r}\right)<1 .
\end{array}\right.
$$

(10) If $1 / 2<\beta \leq 1$ and $n_{0}=\left(\beta / 1-\alpha_{c}\right)$, then $R^{*}(x)=$ $R(x) I_{\left\{x>a_{c}\right\}}$, where $R(x)$ is an increasing 1-Lipschitz continuous function such that $R^{*}(x) \in \mathscr{F}$.
Theorem 6. Under the condition $a_{c}=a_{r}$, the Pareto-optimal reinsurance policies are given as follows:

(1) If $0 \leq \beta<1 / 2$, then 


$$
R^{*}(x)=\left\{\begin{array}{l}
x, \quad \text { when }(1+\theta)\left(1-\alpha_{c}\right)>1, \\
x I_{\left\{x \leq a_{c}\right\}}+R(x) I_{\left\{x>a_{c}\right\}}, \quad \text { when }(1+\theta)\left(1-\alpha_{c}\right)=1, \\
0, \quad \text { when } S_{X}(0) \leq \theta^{*} \text { and }(1+\theta)\left(1-\alpha_{c}\right)<1, \\
x \wedge \operatorname{VaR}_{(\theta / 1+\theta)}(X), \quad \text { when } S_{X}(0)>\theta^{*} \text { and }(1+\theta)\left(1-\alpha_{c}\right)<1,
\end{array}\right.
$$

where $R(x)$ is an increasing 1-Lipschitz continuous function such that $R^{*}(x) \in \mathscr{F}$.

(2) If $\beta=1 / 2$, the objective function is zero and the problem is trivial.
(3) If $1 / 2<\beta \leq 1$, then

$$
R^{*}(x)=\left\{\begin{array}{l}
0, \quad \text { when }(1+\theta)\left(1-\alpha_{c}\right)>1 \\
R(x) I_{\left\{x>a_{c}\right\}}, \quad \text { when }(1+\theta)\left(1-\alpha_{c}\right)=1, \\
x, \quad \text { when } S_{X}(0) \leq \theta^{*} \text { and }(1+\theta)\left(1-\alpha_{c}\right)<1, \\
\left(x-\operatorname{VaR}_{(\theta / 1+\theta)}(X)\right)_{+}, \quad \text { when } S_{X}(0)>\theta^{*} \text { and }(1+\theta)\left(1-\alpha_{c}\right)<1,
\end{array}\right.
$$

where $R(x)$ is an increasing 1-Lipschitz continuous function such that $R^{*}(x) \in \mathscr{F}$.

Remark 1. By comparing our results with those in [19], we would like to point out the relationship between the two articles. First, Cai et al. [19] give the explicit forms of the Pareto-optimal reinsurance contracts under the expected value premium principle by the construction method. In our paper, we use the two-stage optimisation procedure. This technique is intuitive and applicable when the expected value premium principle is replaced by other premium principles. Using this technique, we extend the results in [19] under the TVaR premium principle. Second, under the expected value premium principle, Cai et al. [19] derived the optimal ceded loss functions without considering the relationship between $S_{X}(0)$ and 1 in their Theorems 1 and 2 . However, we discuss the relationship between them and derive different optimal ceded functions from theirs in the case $S_{X}(0)<1$. By comparison, we find that our result is more reasonable.

\section{Numerical Examples}

In this section, we give two numerical examples to illustrate the applications of the results obtained in previous sections.

Example 1 (TVaR principle). Assume that the loss variable $X$ is exponentially distributed with the survival function $S_{X}(x)=e^{-0.001 x}$. In this section, we assume $\theta=0.2$ and $\alpha=0.2$; then $a=223.1$. Using the results in Theorems 1, 3, and 3 , we have the following cases.
Case 1. $\alpha_{c}=0.95$ and $\alpha_{r}=0.99$. In this case, $a=223.1$, $a_{c}=2995.7, \quad a_{r}=4605.2, \quad \operatorname{TVaR}_{\alpha_{c}}(X)=3995.7, \quad$ and $\operatorname{TVaR}_{\alpha_{r}}(X)=5605.2$. The optimal ceded loss function $R^{*}(x)$ is shown in Table 1 , and the various key values of $R^{*}(x)$ are shown in Table 2 .

From Table 1, we know that the optimal reinsurance policy depends on the combining coefficient $\beta$. From Table 2 , obviously, with the increase in the weight coefficient $\beta$, the loss of the insurer $\operatorname{TVaR}_{\alpha_{c}}\left(M_{R^{*}}\right)$ is decreasing while the loss of the reinsurer $\operatorname{TVaR}_{\alpha_{r}}\left(N_{R^{*}}\right)$ and the mean premium $E\left(\pi\left(R^{*}\right)\right)$ are increasing, especially more intuitive when $\beta \in(0.5,0.8419)$. Note that we ignore the key values at the endpoints 0.5 and 0.8419 because the Paretooptimal reinsurance policy at endpoints 0.5 and 0.8419 is uncertain.

Case 2. $\alpha_{c}=0.99$ and $\alpha_{r}=0.95$.

In this case, $a=223.1, a_{c}=4605.2, a_{r}=2995.7$, $\operatorname{TVaR}_{\alpha_{c}}(X)=5605.2$, and $\operatorname{TVaR}_{\alpha_{r}}(X)=3995.7$. The optimal ceded loss function $R^{*}(x)$ is shown in Table 3 , and the various key values of $R^{*}(x)$ are shown in Table 4 .

Case 3. $\alpha_{c}=\alpha_{r}=0.95$.

In this case, $a=223.1, a_{c}=a_{r}=2995.7$, and $\operatorname{TVaR}_{\alpha_{c}}(X)=\operatorname{TVaR}_{\alpha_{r}}(X)=3995.7$. The optimal ceded loss function $R^{*}(x)$ is shown in Table 5 , and the various key values of $R^{*}(x)$ are shown in Table 6 .

Remark 2. Under the expected value premium, assume that the loss variable $X$ is exponentially distributed with the survival function $S_{X}(x)=e^{-0.001 x}$ and $\theta=0.2$. Using the results in Theorems 4, 5, and 6 we get the same results as in [19]. 
TABLE 1: $R^{*}(x)$ with $\alpha_{c}<\alpha_{r}$ under exponential distribution.

\begin{tabular}{lc}
\hline$\beta \in[0,0.5)$ & $R^{*}(x)=x \wedge 405.5$ \\
$\beta=0.5$ & $R^{*}(x)$ is unspecified, $223.1 \leq x \leq 2995.7$ \\
$\beta \in(0.5,0.8419)$ & $R^{*}(x)=u_{1}, x>2995.7, \forall u_{1} \in[223.1,2995.7]$ \\
$\beta=0.8419$ & $R^{*}(x)=\left((x-405.5)_{+} \wedge\left(\mathrm{VaR}_{s(\beta)}(X)-405.5\right)\right), \forall \operatorname{VaR}_{s(\beta)}(X) \in(2995.7,4605.2)$ \\
$\beta \in(0.8419,1]$ & $R^{*}(x)=(x-405.5)_{+}$ \\
\hline
\end{tabular}

TABLE 2: Various key values of $R^{*}(x)$ with $\alpha_{c}<\alpha_{r}$ under exponential distribution.

\begin{tabular}{lccc}
\hline & $\operatorname{TVaR}_{\alpha_{c}}\left(M_{R^{*}}\right)$ & $\operatorname{TVaR}_{\alpha_{r}}\left(N_{R^{*}}\right)$ & $E\left(\pi\left(R^{*}\right)\right)$ \\
\hline$\beta \in[0,0.5)$ & 4058 & -62.3 & 467.8 \\
$\beta \in(0.5,0.8419)$ & $(2330.5 \downarrow 1590.5)$ & $(1665.2 \uparrow 33214.7)$ & $(925 \uparrow 985)$ \\
$\beta \in(0.8419,1]$ & 1405.5 & 4199.7 & 1000 \\
\hline
\end{tabular}

TABLE 3: $R^{*}(x)$ with $\alpha_{c}>\alpha_{r}$ under exponential distribution.

\begin{tabular}{lc}
\hline$\beta \in[0,0.1581)$ & $R^{*}(x)=x \wedge 405.5$ \\
$\beta=0.1581$ & $R^{*}(x)=x \wedge 405.5,223.1 \leq x \leq 4605.2, R^{*}(x)$ is unspecified, $x>4605.2$ \\
$\beta \in(0.1581,0.5)$ & $R^{*}(x)=x \wedge 405.5+\left(x-\operatorname{VaR}_{t(\beta)}(X)\right)_{+}, \forall \operatorname{VaR}_{t(\beta)}(X) \in(2995.7,4605.2)$ \\
$\beta=0.5$ & $R^{*}(x)$ is unspecified, 223.1 $\leq x \leq 4605.2$ \\
$\beta \in(0.5,1]$ & $R^{*}(x)=x-2995.7+u_{2}, x>4605.2, \forall u_{2} \in[223.1,2995.7]$ \\
\hline
\end{tabular}

TABLE 4: Various key values of $R^{*}(x)$ with $\alpha_{c}>\alpha_{r}$ under exponential distribution.

\begin{tabular}{lccc}
\hline & $\operatorname{TVaR}_{\alpha_{c}}\left(M_{R^{*}}\right)$ & $\operatorname{TVaR}_{\alpha_{r}}\left(N_{R^{*}}\right)$ & $E\left(\left(\pi\left(R^{*}\right)\right)\right.$ \\
\hline$\beta \in[0,0.1581)$ & 5667.5 & -62.3 & 467.8 \\
$\beta \in(0.1581,0.5)$ & $(4682.5 \downarrow 3133)$ & $(122.7 \uparrow 862.7)$ & $(482.8 \uparrow 542.8)$ \\
$\beta \in(0.5,1]$ & 1405.5 & 2590.2 & 1000 \\
\hline
\end{tabular}

TABLE 5: $R^{*}(x)$ with $\alpha_{c}=\alpha_{r}$ under exponential distribution.

\begin{tabular}{lc}
\hline$\beta \in[0,0.5)$ & $R^{*}(x)=x \wedge 405.5$ \\
$\beta=0.5$ & $R^{*}(x)$ is unspecified \\
$\beta \in(0.5,1]$ & $R^{*}(x)=(x-405.5)_{+}$ \\
\hline
\end{tabular}

TABLE 6: Various key values of $R^{*}(x)$ with $\alpha_{c}=\alpha_{r}$ under exponential distribution.

\begin{tabular}{lccc}
\hline & $\mathrm{TVaR}_{\alpha_{c}}\left(M_{R^{*}}\right)$ & $\mathrm{TVaR}_{\alpha_{r}}\left(N_{R^{*}}\right)$ & $E\left(\pi\left(R^{*}\right)\right)$ \\
\hline$\beta \in[0,0.5)$ & 4058 & -62.3 & 467.8 \\
$\beta=0.5$ & $(4058 \downarrow 1405.5)$ & $(-62.3 \uparrow 2590.2)$ & $(467.8 \uparrow 1000)$ \\
$\beta \in(0.5,1]$ & 1405.5 & 2590.2 & 1000 \\
\hline
\end{tabular}

Example 2 (expected value premium principle). Assume $\theta=$ 0.2 and the loss variable $X$ with the survival function:

$$
S_{X}(x)= \begin{cases}1, & x<0 \\ 0.25, & x=0 \\ 0.75 e^{-0.001 x}, & x>0\end{cases}
$$

Using the results in Theorems 4, 5, and 6, we have the following cases.
Case 4. $\alpha_{c}=0.95$ and $\alpha_{r}=0.99$. In this case, $a_{c}=2708.1$, $a_{r}=4317.5, \quad \operatorname{TVaR}_{\alpha_{c}}(X)=3708.1, \quad$ and $\operatorname{TVaR}_{\alpha_{r}}(X)=$ 5317.5. The optimal ceded loss function $R^{*}(x)$ is shown in Table 7 , and the various key values of $R^{*}(x)$ are shown in Table 8.

Case 5. $\alpha_{c}=0.99$ and $\alpha_{r}=0.95$.

In this case, $a_{c}=4317.5, a_{r}=2708.1, \operatorname{TVaR} \alpha_{c}(X)=$ 5317.5, and $\operatorname{TVaR}_{\alpha_{r}}(X)=3708.1$. The optimal ceded loss function $R^{*}(x)$ is shown in Table 9, and the various key values of $R^{*}(x)$ are shown in Table 10 .

Case 6. $\alpha_{c}=\alpha_{r}=0.95$.

In this case, $a_{c}=a_{r}=2708.1$ and $\operatorname{TVaR}_{\alpha_{c}}(X)=$ $\operatorname{TVaR}_{\alpha_{r}}(X)=3708.1$. The optimal ceded loss function $R^{*}(x)$ is shown in Table 11, and the various key values of $R^{*}(x)$ are shown in Table 12 .

It is worth mentioning that the distribution in Example 2 is not applicable in [19], and it violates the meaning of the ceded loss function. In addition, note that the parameter $\beta$ and the confidence levels of TVaRs have significant influences on the Pareto-optimal contracts. If $\beta$ is small, the weight of the reinsurer is larger than the insurer, and then 
TABLE 7: $R^{*}(x)$ with $\alpha_{c}<\alpha_{r}$.

\begin{tabular}{lc}
\hline$\beta \in[0,0.5)$ & $R^{*}(x)=0$ \\
$\beta=0.5$ & $R^{*}(x)$ is unspecified, $x \leq 2708.1$ \\
$\beta \in(0.5,0.84)$ & $R^{*}(x)=u_{3}, x>2708.1, \forall u_{3} \in[0,2708.1]$ \\
$\beta=0.84$ & $R^{*}(x)=\min \left\{x, \operatorname{VaR}_{s_{0}(\beta)}(X)\right\}, \forall \operatorname{VaR}_{s_{0}(\beta)}(X) \in(2708.1,4317.5)$ \\
$\beta \in(0.84,1]$ & $R^{*}(x)=x, x \leq 4317.5, R^{*}(x)$ is unspecified, $x>4317.5$ \\
\hline
\end{tabular}

TABLE 8: Various key values of $R^{*}(x)$ with $\alpha_{c}<\alpha_{r}$.

\begin{tabular}{lccc}
\hline & $\mathrm{TVaR}_{\alpha_{c}}\left(M_{R^{*}}\right)$ & $\mathrm{TVaR}_{\alpha_{r}}\left(N_{R^{*}}\right)$ & $E\left(\pi\left(R^{*}\right)\right)$ \\
\hline$\beta \in[0,0.5)$ & 3708.1 & 0 & 0 \\
$\beta \in(0.5,0.84)$ & $(1840 \downarrow 1088)$ & $(1868.1 \uparrow 3429.5)$ & $(840 \uparrow 888)$ \\
$\beta \in(0.84,1]$ & 900 & 4417.5 & 900 \\
\hline
\end{tabular}

TABLE 9: $R^{*}(x)$ with $\alpha_{c}>\alpha_{r}$.

\begin{tabular}{lc}
\hline$\beta \in[0,0.1599)$ & $R^{*}(x)=0$ \\
$\beta=0.1599$ & $R^{*}(x)=0, x \leq 4317.5$ \\
$\beta \in(0.1599,0.5)$ & $R^{*}(x)$ is unspecified, $x>4317.5$ \\
$\beta=0.5$ & $R^{*}(x)=\left(x-\operatorname{VaR}_{t_{0}(\beta)}(X)\right)_{+}, \forall \operatorname{VaR}_{t_{0}(\beta)}(X) \in(2708.1,4317.5)$ \\
$\beta \in(0.5,1]$ & $R^{*}(x)$ is unspecified, $x \leq 2708.1$ \\
\hline
\end{tabular}

TABLE 10: Various key values of $R^{*}(x)$ with $\alpha_{c}>\alpha_{r}$.

\begin{tabular}{lccc}
\hline & $\operatorname{TVaR}_{\alpha_{c}}\left(M_{R^{*}}\right)$ & $\mathrm{TVaR}_{\alpha_{r}}\left(N_{R^{*}}\right)$ & $E\left(\pi\left(R^{*}\right)\right)$ \\
\hline$\beta \in[0,0.1599)$ & 5317.5 & 0 & 0 \\
$\beta \in(0.1599,0.5)$ & $(4329.5 \downarrow 2768.1)$ & $(188 \uparrow 940)$ & $(12 \uparrow 60)$ \\
$\beta \in(0.5,1]$ & 900 & 2808.1 & 900 \\
\hline
\end{tabular}

TABLE 11: $R^{*}(x)$ with $\alpha_{c}=\alpha_{r}$.

\begin{tabular}{lc}
\hline$\beta \in[0,0.5)$ & $R^{*}(x)=0$ \\
$\beta=0.5$ & $R^{*}(x)$ is unspecified \\
$\beta \in(0.5,1]$ & $R^{*}(x)=x$ \\
\hline
\end{tabular}

TABLE 12: Various key values of $R^{*}(x)$ with $\alpha_{c}=\alpha_{r}$.

\begin{tabular}{lccc}
\hline & $\mathrm{TVaR}_{\alpha_{c}}\left(M_{R^{*}}\right)$ & $\mathrm{TVaR}_{\alpha_{r}}\left(N_{R^{*}}\right)$ & $E\left(\pi\left(R^{*}\right)\right)$ \\
\hline$\beta \in[0,0.5)$ & 3708.1 & 0 & 0 \\
$\beta=0.5$ & $(3708.1 \downarrow 900)$ & $(0 \uparrow 2808.1)$ & $(0 \uparrow 900)$ \\
$\beta \in(0.5,1]$ & 900 & 2808.1 & 900 \\
\hline
\end{tabular}

the reinsurer bears less losses. Conversely, if $\beta$ is large, the weight of the insurer is larger than the reinsurer, and then the reinsurer bears more losses. If $\alpha_{c}<\alpha_{r}$, which means that the TVaR standard of the reinsurer is higher than the insurer, then the reinsurer bears less losses. If $\alpha_{c}>\alpha_{r}$, which means that the TVaR standard of the insurer is higher than the reinsurer, then the reinsurer bears more losses.

\section{Conclusion}

In this paper, based on the TVaR measure, we show that the Pareto-optimal reinsurance policies must exist for the insurer and the reinsurer under a class of premium principle, such as the net principle, expected value premium principle, TVaR principle, and generalized percentile. Using a twostage optimisation procedure, we derive explicitly the Pareto-optimal reinsurance policies under the TVaR principle. Since the expected value premium principle can be viewed as a special case of the TVaR principle, then letting $\alpha=0$ in the TVaR principle gives Pareto-optimal reinsurance policies for the expected value premium principle. We extend the results in [19]. Compared with the method used in [19], using the two-stage optimisation method to derive the Pareto-optimal strategy is simpler and more intuitive. Furthermore, by comparing the results in [19] with ours, Cai et al. [19] derived the optimal ceded loss functions without considering the relationship between $S_{X}(0)$ and 1, while we discuss the relationship between $S_{X}(0)$ and 1 and derive different optimal ceded functions from theirs in the case $S_{X}(0)<1$.

We also wish to point out that further research on this topic is needed. First, the risk measure TVaR can be generalized to coherent risk measures. Although some papers have been devoted to deriving optimal reinsurance under coherent risk measures, the optimal reinsurance study still lacks of available analyze tools. Since nonlinear expectation is an essential feature of coherent risk measures, maybe we can draw support from nonlinear expectation; research literatures in this regard are [27-31], etc. Second, we can analyze risk with the strategies of dividend and reinsurance. For more references on the dividend, refer to [32-34], etc. Third, in most of the optimal reinsurance problems, it is assumed that the distributions of the insurer's risks are known. However, in practice, only incomplete information on the distributions is available. How to obtain optimal reinsurance contracts with incomplete information is also an interesting topic. An attempt to such a problem is to use the 
statistical methods. For more references on statistical methods, see, e.g., [35-37]. We hope that these important open problems can be addressed in the future research. We also believe that this article will foster further research in this direction.

\section{Appendix}

The proof of Theorem 1.

By (3), the equivalent form of (13) is

$$
\min _{R \in \mathscr{F}}\left\{\frac{(2 \beta-1)(1+\theta)}{1-\alpha} \int_{\alpha}^{\alpha_{c}} R\left(\operatorname{VaR}_{s}(X)\right) \mathrm{d} s+m \int_{\alpha c}^{\alpha_{r}} R\left(\operatorname{VaR}_{s}(X)\right) \mathrm{d} s+\left(m+\frac{1-\beta}{1-\alpha_{r}}\right) \int_{\alpha_{r}}^{1} R\left(\operatorname{VaR}_{s}(X)\right) \mathrm{d} s\right\} .
$$

(1) If $0 \leq \beta<1 / 2$ and $\left(\beta-1 / 1-\alpha_{r}\right)<m$, by Lemma 1 , we get that (A.1) is solved by

$$
R^{*}\left(X ; \xi, \xi_{c}, \xi_{r}\right)= \begin{cases}(X-a+\xi) \wedge \xi_{c}, & a \leq X \leq a_{c} \\ \left(X-a_{c}+\xi_{c}\right) \wedge \xi_{r}, & a_{c}<X \leq a_{r}, \\ \xi_{r}, & X>a_{r},\end{cases}
$$

where $\left(\xi, \xi_{c}, \xi_{r}\right) \in \mathscr{D}_{1}$ and $\mathscr{D}_{1}=\left\{\left(\xi, \xi_{c}, \xi_{r}\right)\right.$ : $0 \leq \xi \leq a ; 0 \leq \xi_{c} \leq a_{c} ; 0 \leq \xi_{r} \leq a_{r} ; 0 \leq \xi_{r}-\xi_{c} \leq a_{r}-a_{c}$; $\left.0 \leq \xi_{c}-\xi \leq a_{c}-a ; 0 \leq \xi_{r}-\xi \leq a_{r}-a\right\}$. Thus,

$$
\begin{aligned}
& \operatorname{TVaR}_{\alpha}\left(R^{*}\left(X ; \xi, \xi_{c}, \xi_{r}\right)\right)=\xi+\frac{1}{1-\alpha} \int_{a}^{a-\xi+\xi_{c}} S_{X}(x) \mathrm{d} x+\frac{1}{1-\alpha} \int_{a_{c}}^{a_{c}-\xi_{c}+\xi_{r}} S_{X}(x) \mathrm{d} x, \\
& \operatorname{TVaR}_{\alpha_{c}}\left(\left(R^{*}\left(X ; \xi, \xi_{c}, \xi_{r}\right)\right)=\xi_{c}+\frac{1}{1-\alpha_{c}} \int_{a_{c}}^{a_{c}-\xi_{c}+\xi_{r}} S_{X}(x) \mathrm{d} x,\right. \\
& \operatorname{TVaR}_{\alpha_{r}}\left(R^{*}\left(X ; \xi, \xi_{c}, \xi_{r}\right)\right)=\xi_{r} .
\end{aligned}
$$

Define $H\left(R^{*}\right)=H_{1}\left(\xi, \xi_{c}, \xi_{r}\right)$ in this case; then the second-stage optimisation problem is reduced to minimize $H_{1}$. Note that $\left(\partial H_{1} / \quad \partial \xi_{r}\right)=1-\beta+$ $\left[((2 \beta-1)(1+\theta) / 1-\alpha)-\left(\beta / 1-\alpha_{c}\right)\right] S_{X}\left(a_{c}-\xi_{c}+\xi_{r}\right)$, and it is increasing in $\xi_{r}$ on $\left[\xi_{c}, a_{r}-a_{c}+\xi_{c}\right]$ since $m<0$.
(1) When $(1+\theta)\left(1-\alpha_{c}\right)>1-\alpha$, we have $\left.\left(\partial H_{1} / \partial \xi_{r}\right)\right|_{\xi_{r}=\xi_{c}}<0$. Since $m+\left(1-\beta / 1-\alpha_{r}\right)>0$, then we obtain $\left.\left(\partial H_{1} / \partial \xi_{r}\right)\right|_{\xi_{r}=a_{r}-a_{c}+\xi_{c}}>0$. So, $H_{1}$ attains its minimum value at $\xi_{r}^{* c}=\operatorname{VaR}_{s(\beta)}(X)-$ $a_{c}+\xi_{c}$. Note that

$$
\begin{aligned}
H_{1}\left(\xi, \xi_{c}, \xi_{r}^{*}\right)= & -\beta \xi_{c}-\frac{\beta}{1-\alpha_{c}} \int_{a_{c}}^{\operatorname{VaR}_{s(\beta)}(X)} S_{X}(x) \mathrm{d} x+(1-\beta)\left(\operatorname{VaR}_{s(\beta)}(X)-a_{c}+\xi_{c}\right) \\
& +(2 \beta-1)(1+\theta)\left(\xi+\frac{1}{1-\alpha}\left(\int_{a}^{a-\xi+\xi_{c}} S_{X}(x) \mathrm{d} x+\int_{a_{c}}^{\operatorname{VaR}_{s(\beta)}(X)} S_{X}(x) \mathrm{d} x\right)\right),
\end{aligned}
$$

and $\left(\partial H_{1} / \partial \xi_{c}\right)=(2 \beta-1)\left[(1+\theta / 1-\alpha) S_{X}\left(a-\xi_{+}\right.\right.$ $\left.\left.\xi_{c}\right)-1\right]$, so $\left(\partial H_{1} / \partial \xi_{c}\right)$ is increasing in $\xi_{c}$ on $\left[\xi, a_{c}-a+\xi\right]$. Since $\left.\left(\partial H_{1} / \partial \xi_{c}\right)\right|_{\xi_{c}=a_{c}-a+\xi}<0$, then $H_{1}$ attains its minimum value at $\xi_{c}^{*} \stackrel{a_{c}}{=} a_{c}-a+\xi$. Furthermore, $\left(\partial H_{1} / \partial \xi\right)=(2 \beta-1) \theta<0$ always holds, and so $H_{1}$ attains its minimum value at $\xi^{*}=a$. In conclusion, $R^{*}(x)=x \wedge \operatorname{VaR}_{s(\beta)}(X)$.

(2) When $(1+\theta)\left(1-\alpha_{c}\right) \leq 1-\alpha$, we have $\left.\left(\partial H_{1} / \partial \xi_{r}\right)\right|_{\xi_{k}=\xi_{c}} \geq 0$, so $H_{1}$ attains its minimum value at $\xi_{r}^{*}=\xi_{c}$. Note that $H_{1}\left(\xi, \xi_{c}, \xi_{r}^{*}\right)=(1-$ 
$2 \beta) \xi_{c}+(2 \beta-1)(1+\theta)\left(\xi+1 / 1-\alpha \int_{a}^{a-\xi+\xi_{c}} S_{X}(x) \mathrm{d} x\right)$ and $\left(\partial H_{1} / \partial \xi_{c}\right)=(2 \beta-1)\left[(1+\theta / 1-\alpha) S_{X}(a-\xi+\right.$ $\left.\left.\xi_{c}\right)-1\right]$; then $\left(\partial H_{1} / \partial \xi_{c}\right)$ is increasing in $\xi_{c}$ on $\left[\xi, a_{c}-\right.$ $a+\xi]$ since $\left.\left(\partial H_{1} / \partial \xi_{c}\right)\right|_{\xi_{c}=\xi}<0$ and $\left(\partial H_{1} /\right.$ $\left.\partial \xi_{c}\right)\left.\right|_{\xi_{c}=a_{c}-a+\xi} \geq 0$.

When $\left.\left(\partial H_{1} / \partial \xi_{c}\right)\right|_{\xi_{c}=a_{c}-a+\xi}=0$, then $H_{1}$ attains its minimum value at $\xi_{c}^{*}=a_{c}-a+\xi$ and $\xi^{*}=a$. Therefore, $R^{*}(x)=x \wedge a_{c}$.

When $\left.\left(\partial H_{1} / \partial \xi_{c}\right)\right|_{\xi_{c}=a_{c}-a+\xi}>0, H_{1}$ attains its minimum value at $\xi_{c}^{*}=\operatorname{VaR}_{(\theta+\alpha / 1+\theta)}(X)-a+\xi$ and $\xi^{*}=a$. Therefore, $R^{*}(x)=x \wedge \operatorname{VaR}_{(\theta+\alpha / 1+\theta)}(X)$.

Note that $\operatorname{VaR}_{(\theta+\alpha / 1+\theta)}(X)=a_{c}$ if $\left(\partial H_{1} /\right.$ $\left.\partial \xi_{c}\right)\left.\right|_{\xi_{c}=a_{c}-a+\xi}=0$. Therefore, $\quad R^{*}(x)=x \wedge$ $\operatorname{VaR}_{(\theta+\alpha / 1+\theta)}(X)$ when $(1+\theta)\left(1-\alpha_{c}\right) \leq 1-\alpha$.
(2) If $0 \leq \beta<1 / 2$ and $m<\left(\beta-1 / 1-\alpha_{r}\right)$, by Lemma 1 , we get that (A.1) is solved by

$$
R^{*}\left(X ; \xi, \xi_{c}, \xi_{r}\right)= \begin{cases}(X-a+\xi) \wedge \xi_{c}, & a \leq X \leq a_{c}, \\ \left(X-a_{c}+\xi_{c}\right) \wedge \xi_{r}, & a_{c}<X \leq a_{r}, \\ X-a_{r}+\xi_{r}, & X>a_{r},\end{cases}
$$

where $\left(\xi, \xi_{c}, \xi_{r}\right) \in \mathscr{D}_{1}$. Therefore,

$$
\begin{aligned}
& \operatorname{TVaR}_{\alpha}\left(R^{*}\left(X ; \xi, \xi_{c}, \xi_{r}\right)\right)=\xi+\frac{1}{1-\alpha} \int_{a}^{a-\xi+\xi_{c}} S_{X}(x) \mathrm{d} x+\frac{1}{1-\alpha} \int_{a_{c}}^{a_{c}-\xi_{c}+\xi_{r}} S_{X}(x) \mathrm{d} x+\frac{1}{1-\alpha} \int_{a_{r}}^{X_{F}} S_{X}(x) \mathrm{d} x, \\
& \operatorname{TVaR}_{\alpha_{c}}\left(R^{*}\left(X ; \xi, \xi_{c}, \xi_{r}\right)\right)=\xi_{c}+\frac{1}{1-\alpha_{c}} \int_{a_{c}}^{a_{c}-\xi_{c}+\xi_{r}} S_{X}(x) \mathrm{d} x+\frac{1}{1-\alpha_{c}} \int_{a_{r}}^{X_{F}} S_{X}(x) \mathrm{d} x, \\
& \operatorname{TVaR}_{\alpha_{r}}\left(R^{*}\left(X ; \xi, \xi_{c}, \xi_{r}\right)\right)=\xi_{r}+\frac{1}{1-\alpha_{r}} \int_{a_{r}}^{X_{F}} S_{X}(x) \mathrm{d} x .
\end{aligned}
$$

Then,

$$
\begin{aligned}
H\left(R^{*}\right):= & H_{2}\left(\xi, \xi_{c}, \xi_{r}\right) \\
= & -\beta \xi_{c}+(1-\beta) \xi_{r}+(2 \beta-1)(1+\theta) \xi+m \int_{a_{c}}^{a_{c}-\xi_{c}+\xi_{r}} S_{X}(x) \mathrm{d} x \\
& +\frac{(2 \beta-1)(1+\theta)}{1-\alpha} \int_{a}^{a-\xi+\xi_{c}} S_{X}(x) \mathrm{d} x+\left(m+\frac{1-\beta}{1-\alpha_{r}}\right) \int_{a_{r}}^{X_{F}} S_{X}(x) \mathrm{d} x,
\end{aligned}
$$

and $H_{2}$ attains its minimum value at $\left(\xi^{*}, \xi_{c}^{*}, \xi_{r}^{*}\right)=$ $\left(a, a_{c}, a_{r}\right)$ in this case. Therefore, $R^{*}(x)=x$.

(3) If $0 \leq \beta<1 / 2$ and $m=\left(\beta-1 / 1-\alpha_{r}\right)$, by Lemma 1 , we get that (A.1) is solved by

$$
R^{*}\left(X ; \xi, \xi_{c}, \xi_{r}\right)= \begin{cases}(X-a+\xi) \wedge \xi_{c}, & a \leq X \leq a_{c}, \\ \left(X-a_{c}+\xi_{c}\right) \wedge \xi_{r}, & a_{c}<X \leq a_{r}, \\ R(x), & X>a_{r},\end{cases}
$$


where $R(x)$ is an increasing 1-Lipschitz continuous function. Therefore,

$$
\begin{aligned}
& \operatorname{TVaR}_{\alpha}\left(R^{*}\left(X ; \xi, \xi_{c}, \xi_{r}\right)\right)=\xi+\frac{1}{1-\alpha} \int_{a}^{X_{F}} \mathrm{P}\left(R^{*}\left(X ; \xi, \xi_{c}, \xi_{r}\right)>x\right) \mathrm{d} x, \\
& \operatorname{TVaR}_{\alpha_{c}}\left(R^{*}\left(X ; \xi, \xi_{c}, \xi_{r}\right)\right)=\xi_{c}+\frac{1}{1-\alpha_{c}} \int_{a_{c}}^{X_{F}} \mathrm{P}\left(R^{*}\left(X ; \xi, \xi_{c}, \xi_{r}\right)>x\right) \mathrm{d} x, \\
& \operatorname{TVaR}_{\alpha_{r}}\left(R^{*}\left(X ; \xi, \xi_{c}, \xi_{r}\right)\right)=\xi_{r}+\frac{1}{1-\alpha_{r}} \int_{a_{r}}^{X_{F}} \mathrm{P}\left(R^{*}\left(X ; \xi, \xi_{c}, \xi_{r}\right)>x\right) \mathrm{d} x .
\end{aligned}
$$

In this case,

$$
\begin{aligned}
H\left(R^{*}\right) & :=H_{3}\left(\xi, \xi_{c}, \xi_{r}\right) \\
& =-\beta \xi_{c}+(1-\beta) \xi_{r}+(2 \beta-1)(1+\theta) \xi+m \int_{a_{c}}^{a_{c}-\xi_{c}+\xi_{r}} S_{X}(x) \mathrm{d} x+\frac{(2 \beta-1)(1+\theta)}{1-\alpha} \int_{a}^{a-\xi+\xi_{c}} S_{X}(x) \mathrm{d} x,
\end{aligned}
$$

and $H_{3}$ attains its minimum value at $\left(\xi^{*}, \xi_{c}^{*}, \xi_{r}^{*}\right)=$

$\left(a, a_{c}, a_{r}\right)$. Therefore, $R^{*}(x)=x I_{\left\{a \leq x \leq a_{r}\right\}}+R(x)$

Then,

$I_{\left\{x>a_{r}\right\}}$.

(4) If $\beta=1 / 2$, by Lemma 1 , we get that (A.1) is solved by

$$
R^{*}\left(X ; \xi, \xi_{c}, \xi_{r}\right)= \begin{cases}R(x), & a \leq X \leq a_{c} \\ \left(X-a_{c}+\xi_{c}\right) \wedge \xi_{r}, & a_{c}<X \leq a_{r} \\ \xi_{r}, & X>a_{r}\end{cases}
$$

$$
\begin{aligned}
\operatorname{TVaR}_{\alpha}\left(R^{*}\left(X ; \xi, \xi_{c}, \xi_{r}\right)\right) & =\xi+\frac{1}{1-\alpha} \int_{a}^{X_{F}} \mathrm{P}\left(R^{*}\left(X ; \xi, \xi_{c}, \xi_{r}\right)>x\right) \mathrm{d} x \\
\operatorname{TVaR}_{\alpha_{c}}\left(R^{*}\left(X ; \xi, \xi_{c}, \xi_{r}\right)\right) & =\xi_{c}+\frac{1}{1-\alpha_{c}} \int_{a_{c}}^{a_{c}-\xi_{c}+\xi_{r}} S_{X}(x) \mathrm{d} x \\
\operatorname{TVaR}_{\alpha_{r}}\left(R^{*}\left(X ; \xi, \xi_{c}, \xi_{r}\right)\right) & =\xi_{r}, \\
H\left(R^{*}\right) & :=H_{4}\left(\xi, \xi_{c}, \xi_{r}\right) \\
& =\frac{1}{2} \xi_{c}+\frac{1}{2} \xi_{r}+\frac{1}{2\left(1-\alpha_{c}\right)} \int_{a_{c}}^{a_{c}-\xi_{c}+\xi_{r}} S_{X}(x) \mathrm{d} x
\end{aligned}
$$

It is easy to see that $H_{4}$ attains its minimum value at $\left(\xi^{*}, \xi_{c}^{*}, \xi_{r}^{*}\right)=\left(\xi, u_{1}, u_{1}\right)$, where $u_{1} \in\left[a, a_{c}\right]$. Therefore, $R^{*}(x)=R(x) I_{\left\{a \leq x \leq a_{c}\right\}}+u_{1} I_{\left\{x>a_{c}\right\}}$.
(5) If $1 / 2<\beta \leq 1$ and $m>0$, the coefficients of the three integrals in (A.1) are all positive, obviously $R^{*}(x)=0$.

(6) If $1 / 2<\beta<1$ and $\left(\beta-1 / 1-\alpha_{r}\right)<m<0$, by Lemma 1 , we get that (A.1) is solved by 
Then,

$R^{*}\left(X ; \xi, \xi_{c}, \xi_{r}\right)= \begin{cases}\xi+\left(X-a_{c}+\xi_{c}-\xi\right)_{+}, & a \leq X \leq a_{c}, \\ \left(X-a_{c}+\xi_{c}\right) \wedge \xi_{r}, & a_{c}<X \leq a_{r}, \\ \xi_{r}, & X>a_{r} .\end{cases}$

$H\left(R^{*}\right):=H_{5}\left(\xi, \xi_{c}, \xi_{r}\right)$

$$
=-\beta \xi_{c}+(1-\beta) \xi_{r}-\frac{\beta}{1-\alpha_{c}} \int_{a_{c}}^{a_{c}-\xi_{c}+\xi_{r}} S_{X}(x) \mathrm{d} x+(2 \beta-1)(1+\theta)\left(\xi+\frac{1}{1-\alpha} \int_{a_{c}-\xi_{c}+\xi}^{a_{c}-\xi_{c}+\xi_{r}} S_{X}(x) \mathrm{d} x\right) .
$$

Note that $\left(\partial H_{5} / \partial \xi_{r}\right)=1-\beta+[((2 \beta-1)(1+\theta) / 1-$ $\left.\alpha)-\left(\beta / 1-\alpha_{c}\right)\right] S_{X}\left(a_{c}-\xi_{c}+\xi_{r}\right)$ is increasing in $\xi_{r}$ on $\left[\xi_{c}, a_{r}-a_{c}+\xi_{c}\right]$.

(1) When $(1+\theta)\left(1-\alpha_{c}\right) \geq 1-\alpha, H_{5}$ attains its minimum value at $\left(\xi^{*}, \xi_{c}^{*}, \xi_{r}^{*}\right)=(0,0,0)$. Therefore, $R^{*}(x)=0$.

(2) When $(1+\theta)\left(1-\alpha_{c}\right)<1-\alpha, H_{5}$ attains its minimum value at $\left(\xi^{*}, \xi_{c}^{*}, \xi_{r}^{*}\right)=\left(0, a_{c}-\right.$ $\left.\operatorname{VaR}_{(\theta+\alpha / 1+\theta)}(X), \operatorname{VaR}_{S(\beta)}(X)-\operatorname{VaR}_{(\theta+\alpha / 1+\theta)}(X)\right)$. Therefore, $\quad R^{*}(x)=\left(x-\operatorname{VaR}_{(\theta+\alpha / 1+\theta)}(X)\right)_{+}$ $\wedge\left(\operatorname{VaR}_{S(\beta)}(X)-\operatorname{VaR}{ }_{(\theta+\alpha / 1+\theta)}(X)\right)$.
(7) If $1 / 2<\beta \leq 1$ and $m<\left(\beta-1 / 1-\alpha_{r}\right)$, then (A.1) is solved by

$$
R^{*}\left(X ; \xi, \xi_{c}, \xi_{r}\right)= \begin{cases}\xi+\left(X-a_{c}+\xi_{c}-\xi\right)_{+}, & a \leq X \leq a_{c} \\ \left(X-a_{c}+\xi_{c}\right) \wedge \xi_{r}, & a_{c}<X \leq a_{r}, \\ x-a_{r}+\xi_{r}, & X>a_{r} .\end{cases}
$$

Note that

$$
\begin{aligned}
H\left(R^{*}\right):= & H_{6}\left(\xi, \xi_{c}, \xi_{r}\right) \\
= & -\beta \xi_{c}-\frac{\beta}{1-\alpha_{c}} \int_{a_{c}}^{a_{c}-\xi_{c}+\xi_{r}} S_{X}(x) \mathrm{d} x+(1-\beta) \xi_{r}+(2 \beta-1)(1+\theta) \xi+\frac{(2 \beta-1)(1+\theta)}{1-\alpha} \int_{a_{c}-\xi_{c}+\xi}^{a_{c}-\xi_{c}+\xi_{r}} S_{X}(x) \mathrm{d} x \\
& +(m+1-\beta) \int_{a_{r}}^{X_{F}} S_{X}(x) \mathrm{d} x,
\end{aligned}
$$

then $H_{6}$ attains its minimum value at $\left(\xi^{*}, \xi_{c}^{*}, \xi_{r}^{*}\right)=$ $\left(0, a_{c}-\operatorname{VaR}_{(\theta+\alpha / 1+\theta)}(X), a_{r}-\operatorname{VaR}_{(\theta+\alpha / 1+\theta)}(X)\right)$.

Therefore, $R^{*}(x)=\left(x-\operatorname{VaR}_{(\theta+\alpha / 1+\theta)}(X)\right)_{+}$.

(8) If $1 / 2<\beta<1$ and $m=\left(\beta-1 / 1-\alpha_{r}\right)$, then (A.1) is solved by

$$
R^{*}\left(X ; \xi, \xi_{c}, \xi_{r}\right)= \begin{cases}\xi+\left(X-a_{c}+\xi_{c}-\xi\right)_{+}, & a \leq X \leq a_{c}, \\ \left(X-a_{c}+\xi_{c}\right) \wedge \xi_{r}, & a_{c}<X \leq a_{r}, \\ R(x), & X>a_{r} .\end{cases}
$$

We obtain $\quad R^{*}(x)=\left(x-\operatorname{VaR}_{(\theta+\alpha / 1+\theta)}(X)\right)_{+}$ $I_{\left\{x \leq a_{r}\right\}}+R(x) I_{\left\{x>a_{r}\right\}}$.

(9) If $1 / 2<\beta<1$ and $m=0$, then (A.1) is solved by

$$
R^{*}\left(X ; \xi, \xi_{c}, \xi_{r}\right)= \begin{cases}\xi+\left(X-a_{c}+\xi_{c}-\xi\right)_{+}, & a \leq X \leq a_{c}, \\ R(x), & a_{c}<X \leq a_{r}, \\ \xi_{r}, & X>a_{r} .\end{cases}
$$

It is easy to get $\xi_{r}^{*}=\xi_{c}^{*}=\xi^{*}=0$, so $R^{*}(x)=0$. (10) If $\beta=1$ and $m=0$, then (A.1) is solved by

$$
R^{*}\left(X ; \xi, \xi_{c}, \xi_{r}\right)= \begin{cases}\xi+\left(X-a_{c}+\xi_{c}-\xi\right)_{+}, & a \leq X \leq a_{c}, \\ R(x), & a_{c}<X \leq a_{r}, \\ R(x), & X>a_{r} .\end{cases}
$$


Obviously, $R^{*}\left(X ; \xi, \xi_{c}, \xi_{r}\right)$ is independent of $\xi_{r}$, and it is easy to get $\xi_{c}^{*}=\xi^{*}=0$, so $R^{*}(x)=R(x) I_{\left\{x>a_{c}\right\}}$, where $R(x)$ is an increasing 1-Lipschitz continuous function such that $R^{*}(x) \in \mathscr{F}$.

The proof of Theorem 2 .

By (3), the equivalent form of (13) is

$$
\begin{aligned}
& \min _{R \in \mathscr{F}}\left\{\frac{(2 \beta-1)(1+\theta)}{1-\alpha} \int_{\alpha}^{\alpha_{r}} R\left(\operatorname{VaR}_{s}(X)\right) \mathrm{d} s\right. \\
& \quad+n \int_{\alpha_{r}}^{\alpha_{c}} R\left(\operatorname{VaR}_{s}(X)\right) \mathrm{d} s+\left(n-\frac{\beta}{1-\alpha_{c}}\right) \int_{\alpha_{c}}^{1} R\left(\operatorname{VaR}_{s}(X)\right) \mathrm{d} s
\end{aligned}
$$

Using the same method as the proof of Theorem 1, we can obtain the desired results, so we omit the proof. It is worth noting that $\left(\xi, \xi_{c}, \xi_{r}\right) \in \mathscr{D}_{2}$ and $\mathscr{D}_{2}=\left\{\left(\xi, \xi_{c}, \xi_{r}\right)\right.$ : $0 \leq \xi \leq a ; 0 \leq \xi_{c} \leq a_{c} ; 0 \leq \xi_{r} \leq a_{r} ; 0 \leq \xi_{c}-\xi_{r} \leq a_{c}-a_{r} ; 0 \leq \xi_{c}-$ $\left.\xi \leq a_{c}-a ; 0 \leq \xi_{r}-\xi \leq a_{r}-a\right\}$.

The proof of Theorem 3 .

By (3), the equivalent form of (13) is

$$
\begin{aligned}
& \min _{R \in \mathscr{F}}\left\{\frac{(2 \beta-1)(1+\theta)}{1-\alpha} \int_{\alpha}^{\alpha_{c}} R\left(\operatorname{VaR}_{s}(X)\right) \mathrm{d} s\right. \\
& \left.\quad+\left[(2 \beta-1)\left(\frac{1+\theta}{1-\alpha}-\frac{1}{1-\alpha_{c}}\right)\right] \int_{\alpha_{c}}^{1} R\left(\operatorname{VaR}_{s}(X)\right) \mathrm{d} s\right\} .
\end{aligned}
$$

Note that $\left(\xi, \xi_{c}\right) \in \mathscr{D}_{3}$ and $\mathscr{D}_{3}=\left\{\left(\xi, \xi_{c}\right): 0 \leq \xi \leq\right.$ $\left.a ; 0 \leq \xi_{c} \leq a_{c} ; 0 \leq \xi_{c}-\xi \leq a_{c}-a\right\}$. Then, the same technique as used in the proof of Theorem 1 yields the results.

\section{Data Availability}

The data used to support the findings of this study are available from the corresponding author upon request.

\section{Conflicts of Interest}

The authors declare that they have no conflicts of interest.

\section{Acknowledgments}

The research was supported by the Project of the Shandong Province Higher Educational Science and Technology Program (J18KA249) and the Social Science Planning Project of Shandong Province (20CTJJ02).

\section{References}

[1] K. Borch, "An attempt to determine the optimum amount of stop loss reinsurance," Transactions of the 16th International Congress of Actuaries, vol. 1, pp. 597-610, 1960.

[2] K. J. Arrow, "Uncertainty and the welfare economics of medical care," American Economic Review, vol. 53, pp. 941973, 1963.
[3] V. R. Young, "Optimal insurance under Wang's premium principle," Insurance: Mathematics and Economics, vol. 25, no. 2, pp. 109-122, 1999.

[4] M. Kaluszka, "Optimal reinsurance under mean-variance premium principles," Insurance: Mathematics and Economics, vol. 28 , no. 1, pp. 61-67, 2001.

[5] M. Kaluszka and A. Okolewski, "An extension of arrow's result on optimal reinsurance contract," Journal of Risk \& Insurance, vol. 75, no. 2, pp. 275-288, 2008.

[6] J. Cai and K. S. Tan, "Optimal retention for a stop-loss reinsurance under the VaR and CTE risk measures," ASTIN Bulletin, vol. 37, no. 1, pp. 93-112, 2007.

[7] J. Cai, K. S. Tan, C. Weng, and Y. Zhang, "Optimal reinsurance under VaR and CTE risk measures," Insurance: Mathematics and Economics, vol. 43, no. 1, pp. 185-196, 2008.

[8] C. Bernard and W. Tian, "Optimal reinsurance arrangements under tail risk measures," Journal of Risk and Insurance, vol. 76, no. 3, pp. 709-725, 2009.

[9] K. C. Cheung, "Optimal reinsurance revisited-a geometric approach,” ASTIN Bulletin, vol. 40, no. 1, pp. 221-239, 2010.

[10] Y. C. Chi and K. S. Tan, "Optimal reinsurance under VaR and CVaR risk measures: a simplified approach," ASTIN Bulletin, vol. 41, pp. 487-509, 2011.

[11] S. Vajda, "Minimum variance reinsurance," ASTIN Bulletin, vol. 2, no. 2, pp. 257-260, 1962.

[12] V. K. Kaishev, "Optimal retention levels, given the joint survival of cedent and reinsurer," Scandinavian Actuarial Journal, vol. 2004, no. 6, pp. 401-430, 2004.

[13] J. Cai, Y. Fang, Z. Li, and G. E. Willmot, "Optimal reciprocal reinsurance treaties under the joint survival probability and the joint profitable probability," Journal of Risk and Insurance, vol. 80, no. 1, pp. 145-168, 2013.

[14] Y. Fang and Z. Qu, "Optimal combination of quota-share and stop-loss reinsurance treaties under the joint survival probability," IMA Journal of Management Mathematics, vol. 25, no. 1, pp. 89-103, 2014.

[15] Y. Fang, G. Cheng, and Z. Qu, "Optimal reinsurance for both an insurer and a reinsurer under general premium principles," AIMS Mathematics, vol. 5, no. 4, pp. 3231-3255, 2020.

[16] J. Cai, C. Lemieux, and F. Liu, "Optimal reinsurance from the perspectives of both an insurer and a reinsurer," ASTIN Bulletin, vol. 46, no. 3, pp. 815-849, 2016.

[17] A. Lo, "A Neyman-Pearson perspective on optimal reinsurance with constraints," ASTIN Bulletin, vol. 47, no. 2, pp. 467-499, 2017.

[18] W. J. Jiang, J. D. Ren, and R. Zitikis, "Optimal reinsurance policies under the VaR risk measure when the interests of both the cedent and the reinsurer are taken into account," Risks, vol. 5, pp. 1-22, 2017a.

[19] J. Cai, H. Liu, and R. Wang, "Pareto-optimal reinsurance arrangements under general model settings," Insurance: Mathematics and Economics, vol. 77, pp. 24-37, 2017.

[20] W. Jiang, H. Hong, and J. Ren, "On Pareto-optimal reinsurance with constraints under distortion risk measures," European Actuarial Journal, vol. 8, no. 1, pp. 215-243, $2017 \mathrm{~b}$.

[21] Y. Fang, X. Wang, H. Liu, and T. Li, "Pareto-optimal reinsurance for both the insurer and the reinsurer with general premium principles," Communications in Statistics-Theory and Methods, vol. 48, no. 24, pp. 6134-6154, 2019.

[22] A. Lo and Z. Tang, "Pareto-optimal reinsurance policies in the presence of individual risk constraints," Annals of Operations Research, vol. 274, no. 1-2, pp. 395-423, 2019. 
[23] Y. Huang and C. Yin, "A unifying approach to constrained and unconstrained optimal reinsurance," Journal of Computational and Applied Mathematics, vol. 360, pp. 1-17, 2019.

[24] A. V. Asimit, A. M. Badescu, and T. Verdonck, "Optimal risk transfer under quantile-based risk measurers," Insurance: Mathematics and Economics, vol. 53, no. 1, pp. 252-265, 2013.

[25] G. Huberman, D. Mayers, and C. W. Smith Jr., "Optimal insurance policy indemnity schedules," The Bell Journal of Economics, vol. 14, no. 2, pp. 415-426, 1983.

[26] V. R. Young, "Premium principles," in Encyclopedia of Actuarial Science, J. Teugels and B. Sundt., Eds., Vol. 3, John Wiley \& Sons, Hoboken, NJ, USA, 2004.

[27] C. Hu, "Strong laws of large numbers for sublinear expectation under controlled 1st moment condition," Chinese Annals of Mathematics, Series B, vol. 39, no. 5, pp. 791-804, 2018.

[28] C. Hu, "Central limit theorems for sub-linear expectation under the Lindeberg condition," Journal of Inequalities and Applications, vol. 2018, no. 1, 2018.

[29] C. Hu, "Weak and strong laws of large numbers for sub-linear expectation," Communications in Statistics-Theory and Methods, vol. 49, no. 2, pp. 430-440, 2019.

[30] C. Hu, "Marcinkiewicz-Zygmund laws of large numbers under sublinear expectation," Mathematical Problems in Engineering, vol. 2020, Article ID 5050973, 11 pages, 2020.

[31] X. J. Shi, R. L. Ji, and Q. Feng, "Representation of filtrationconsistent nonlinear expectation by g-expectation in general framework," Communications in Statistics-Theory and Methods, 2020.

[32] Y. Zhao, P. Chen, and H. Yang, "Optimal periodic dividend and capital injection problem for spectrally positive Lévy processes," Insurance: Mathematics and Economics, vol. 74, pp. 135-146, 2017.

[33] W. Yu, P. Guo, Q. Wang et al., "On a periodic capital injection and barrier dividend strategy in the compound Poisson risk model," Mathematics, vol. 8, no. 4, p. 511, 2020.

[34] X. Peng, W. Su, W. Su, and Z. Zhang, "On a perturbed compound Poisson risk model under a periodic thresholdtype dividend strategy," Journal of Industrial \& Management Optimization, vol. 16, no. 4, pp. 1967-1986, 2020.

[35] H. Y. Wang and Z. Wu, "Eigenvalues of stochastic Hamiltonian systems driven by Poisson process with boundary conditions," Boundary Value Problems, vol. 2017, no. 1, 2017.

[36] X. Wang, Y. Song, and L. Lin, "Handling estimating equation with nonignorably missing data based on SIR algorithm," Journal of Computational and Applied Mathematics, vol. 326, pp. 62-70, 2017.

[37] Q. Zhao, R. J. Karunamuni, and J. J. Wu, "An empirical classification procedure for nonparametric mixture models," Journal of the Korean Statistical Society, vol. 49, pp. 924-952, 2020. 